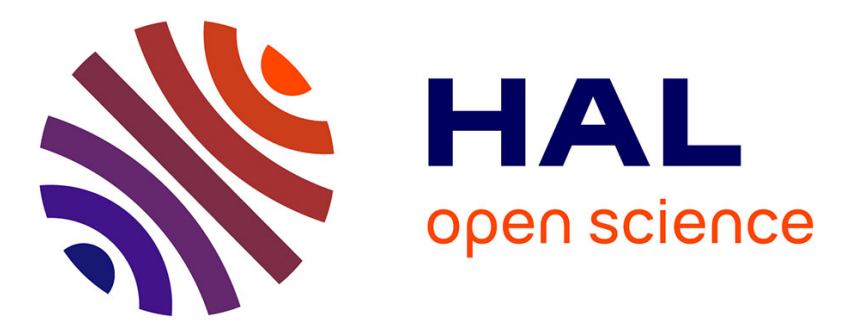

\title{
VO 2 Substrate Effect on the Thermal Rectification of a Far-Field Radiative Diode
}

\author{
I y Forero-Sandoval, J A Chan-Espinoza, Jose Ordonez-Miranda, J J \\ Alvarado-Gil, Frédéric Dumas-Bouchiat, Corinne Champeaux, K Joulain, Y \\ Ezzahri, J Drevillon, C L Gomez-Heredia, et al.
}

\section{To cite this version:}

I y Forero-Sandoval, J A Chan-Espinoza, Jose Ordonez-Miranda, J J Alvarado-Gil, Frédéric DumasBouchiat, et al.. VO 2 Substrate Effect on the Thermal Rectification of a Far-Field Radiative Diode. Physical Review Applied, 2020, 14, 10.1103/physrevapplied.14.034023 . hal-03017598

\section{HAL Id: hal-03017598 \\ https://hal.science/hal-03017598}

Submitted on 21 Nov 2020

HAL is a multi-disciplinary open access archive for the deposit and dissemination of scientific research documents, whether they are published or not. The documents may come from teaching and research institutions in France or abroad, or from public or private research centers.
L'archive ouverte pluridisciplinaire HAL, est destinée au dépôt et à la diffusion de documents scientifiques de niveau recherche, publiés ou non, émanant des établissements d'enseignement et de recherche français ou étrangers, des laboratoires publics ou privés. 


\title{
$\mathrm{VO}_{2}$ Substrate Effect on the Thermal Rectification of a Far-Field Radiative Diode
}

\author{
I.Y. Forero-Sandoval $\odot,{ }^{1,2, *}$ J.A. Chan-Espinoza $\odot,{ }^{2}$ J. Ordonez-Miranda $\odot,{ }^{1, \dagger}$ J.J. Alvarado-Gil, ${ }^{2}$ \\ F. Dumas-Bouchiat $\odot,{ }^{3, \ddagger}$ C. Champeaux $\odot,{ }^{3}$ K. Joulain, ${ }^{1}$ Y. Ezzahri $\odot,{ }^{1}$ J. Drevillon $\odot,{ }^{1}$ \\ C.L. Gomez-Heredia, ${ }^{2}$ and J.A. Ramirez-Rincon ${ }^{2}$ \\ 'Institut Pprime, CNRS, Université de Poitiers, ISAE-ENSMA, Futuroscope Chasseneuil F-86962, France \\ ${ }^{2}$ Applied Physics Department, CINVESTAV-IPN Mérida, Mérida, Yucatán C.P. 97310, México \\ ${ }^{3}$ Université de Limoges, CNRS, IRCER, UMR 7315, Limoges F-87000, France
}

(Received 11 June 2020; revised 5 August 2020; accepted 10 August 2020; published 9 September 2020)

\begin{abstract}
Thermal rectification of far-field heat currents is experimentally demonstrated by exploiting the metalinsulator transition of $\mathrm{VO}_{2}$ driving the significant temperature variations of its emissivity, within a narrow interval of temperatures. This is achieved by measuring remarkable differences on the radiative heat flux between a $\mathrm{VO}_{2}$ film placed in vacuum and in front of a heat fluxmeter, when their temperature difference is reversed. By testing three $\mathrm{VO}_{2}$ films deposited on a substrate of $r$-sapphire, $c$-sapphire, and silicon, the highest rectification factor of $61 \%$ is obtained for the first film operating with a temperature difference of $40^{\circ} \mathrm{C}$ with respect to the fluxmeter. This rectification factor is higher than or comparable to the respective ones reported in the literature for near- or far-field radiative diodes subjected to a temperature difference of $70^{\circ} \mathrm{C}$ between their terminals. This experimental value is consistent with the theoretical one predicted by an analytical expression derived for the maximum rectification factor, as a function of the $\mathrm{VO}_{2}$ emissivity in the metallic and insulating phases, sensor emissivity, and geometrical parameters. The obtained results thus show that the rectification factor of these diodes can be enhanced, while reducing the temperature difference of their terminals, by increasing not only the emissivity variations between the insulating and metallic phases of $\mathrm{VO}_{2}$ films deposited on $r$-sapphire, but also decreasing their emissivity in the metallic phase.
\end{abstract}

DOI: 10.1103/PhysRevApplied.14.034023

\section{INTRODUCTION}

The control of heat currents by means of thermal diodes [1-5], thermal transistors [6-10], thermal logical gates $[11,12]$, and even thermal memories $[13,14]$ has recently attracted significant interest due to its potential applications for processing information with heat. In particular, these thermal diodes are capable of rectifying heat currents when the temperature difference between their two terminals are reversed $[15,16]$. An ideal thermal diode would allow passing heat in one (forward) direction and block it in the opposite (backward) one. In general, the thermal performance of a thermal diode can thus be characterized by the following rectification factor normalized to unity $[3,15]$

$$
R=\frac{|| q_{b}|-| q_{f}||}{\max \left(\left|q_{b}\right| ;\left|q_{f}\right|\right)}
$$

where $q_{b}$ and $q_{f}$ are the heat fluxes in the backward and forward configurations, respectively. Among the different

\footnotetext{
*iyforeos@gmail.com

†jose.ordonez@cnrs.pprime.fr

†rederic.dumas-bouchiat@unilim.fr
}

thermal diodes of conductive [17-20], convective [21,22], and radiative $[23,24]$ heat currents that were proposed, the latter ones generally exhibit higher rectification factors. Furthermore, theoretical models showed that near-field radiative diodes usually yield better thermal rectifications than far-field ones $[10,25]$, due to the significant contribution of surface phonon polaritons involved in the near-field regime $[26,27]$.

Theoretical [3] and experimental [28,29] studies have shown that the metal-insulator transition (MIT) of vanadium dioxide $\left(\mathrm{VO}_{2}\right)$ can efficiently be used to make up thermal diodes. This reversible phase transition occurs at around $68^{\circ} \mathrm{C}[30,31]$ and induces significant changes of the thermal $[29,32,33]$, electrical [34,35], and optical $[28,30,34-36]$ properties of $\mathrm{VO}_{2}$. By using terminals of $\mathrm{Si}$ and $\mathrm{VO}_{2}$ (350-nm-thick film grown on $c$-sapphire) separated by an approximately 140 -nm vacuum gap and exchanging near-field heat with a temperature difference of $70^{\circ} \mathrm{C}$, Fiorino et al. [23] reported a rectification factor lower than $60 \%$. In the far-field regime, on the other hand, Ito et al. [24] found a maximum rectification factor of $66 \%$ by using terminals of fused quartz and $\mathrm{VO}_{2}$ (deposited on a silicon substrate) separated by $1 \mathrm{~mm}$ and operating with a 
temperature difference of $70^{\circ} \mathrm{C}$. These experimental works $[23,24]$ indicate that far-field thermal diodes are not only easier to implement in practice than near-field ones, but also they could yield higher rectification factors, provided that a suitable $\mathrm{VO}_{2}$ terminal is used.

In this work, three $\mathrm{VO}_{2}$ films deposited on substrates of $r$-sapphire, $c$-sapphire, and silicon are tested as one of the terminals of a far-field thermal diode to experimentally assess its rectification factor. The highest rectification factor of $61 \%$ is obtained with $r$-sapphire and a temperature difference of $40^{\circ} \mathrm{C}$ between the diode terminals. Even though this optimal rectification factor is comparable to the ones reported in the literature [23,24], it represents an improvement to them, as it is obtained with a reduction of $30^{\circ} \mathrm{C}$ on the temperature difference of the diode terminals. This significant reduction is the result of the relatively narrow temperature interval spanning the phase transition of the $\mathrm{VO}_{2}$ and $r$-sapphire sample and its implementation is expected to lower the energy required to generate the rectification effect and hence facilitate the operation of radiative thermal diodes. Furthermore, an analytical expression for the maximum rectification factor is also derived, as a function of the $\mathrm{VO}_{2}$ emissivity in the metallic and insulating phases, sensor emissivity, and geometrical parameters.

\section{EXPERIMENTAL SETUP}

Our thermal diode consists of a $\mathrm{VO}_{2}$ film at temperature $T_{\mathrm{VO}_{2}}$ exchanging heat by far-field radiation with a heat-flux sensor (HFS) at temperature $T_{s}$, as shown in Fig. 1(a). Taking into account that the temperature variations of the emissivity $\epsilon_{\mathrm{VO}_{2}}$ of $\mathrm{VO}_{2}$ are driven by its
MIT appearing at the critical temperature $T_{c}$, the temperature interval $\left(T_{\mathrm{VO}_{2}} ; T_{S}\right)$ has to contains $T_{c}$ for capitalizing on the MIT of $\mathrm{VO}_{2}$. Therefore, in the forward configuration, the heat flux $\left(q_{f}\right)$ flows from the $\mathrm{VO}_{2}$ film to the $\operatorname{HFS}\left(T_{\mathrm{VO}_{2}}>T_{s}\right)$ and its value is driven by the relatively low emissivity $\left[\epsilon_{\mathrm{VO}_{2}(m)}\right]$ of $\mathrm{VO}_{2}$ in its metallic phase. In the backward configuration defined by the interchange of the temperature values $\left(T_{\mathrm{VO}_{2}}<T_{s}\right)[15,16]$, on the other hand, the heat flux $\left(q_{b}\right)$ exhibits an opposite direction and is enhanced $\left(q_{b}>q_{f}\right)$ by the emissivity $\left[\epsilon_{\mathrm{VO}_{2}(i)}>\epsilon_{\mathrm{VO}_{2}(m)}\right]$ of $\mathrm{VO}_{2}$ in its insulating phase. The average emissivity $\epsilon_{s}$ of the HFS is considered to be independent of temperature and is determined from the FTIR spectra for wavelengths from 8 to $15 \mu \mathrm{m}$ through the Perkin Elmer Spectrometer (Model Frontier NIR-MED) [36].

The $\mathrm{VO}_{2}$ film is deposited on a substrate $\left(\mathrm{VO}_{2}+\mathrm{S}\right)$ and separated from the HFS by a vacuum gap of $2.3 \mathrm{~mm}$ to radiatively couple them in the far-field regime [37], as shown in Fig. 1(b). The lateral dimensions of the $\mathrm{VO}_{2}$ films and HFS are $A_{\mathrm{VO}_{2}}=1.5 \times 1.5 \mathrm{~cm}^{2}, A_{s}=1 \times 1 \mathrm{~cm}^{2}$, respectively; and their temperatures are controlled with Peltier cells (TED) and Proportional -Integral -Derivative (PID) controllers (Tetech, TC-48-20) attached to them. The temperatures are measured by means of thermistors of 0.9 $\mathrm{mm}$ in diameter (Tetech, MP-2444), placed as shown in Fig. 1(b). The cold face of each Peltier cell is chilled by a flux of water at a mean temperature $T_{w}$. The whole system is placed in a vacuum chamber evacuated with a pressure below $10^{-5}$ Torr to suppress the conductive heat currents through the intracavity air to values smaller than $0.1 \%$ of the blackbody radiation. The linear response of the HFS to heat-flux variations depends on its average
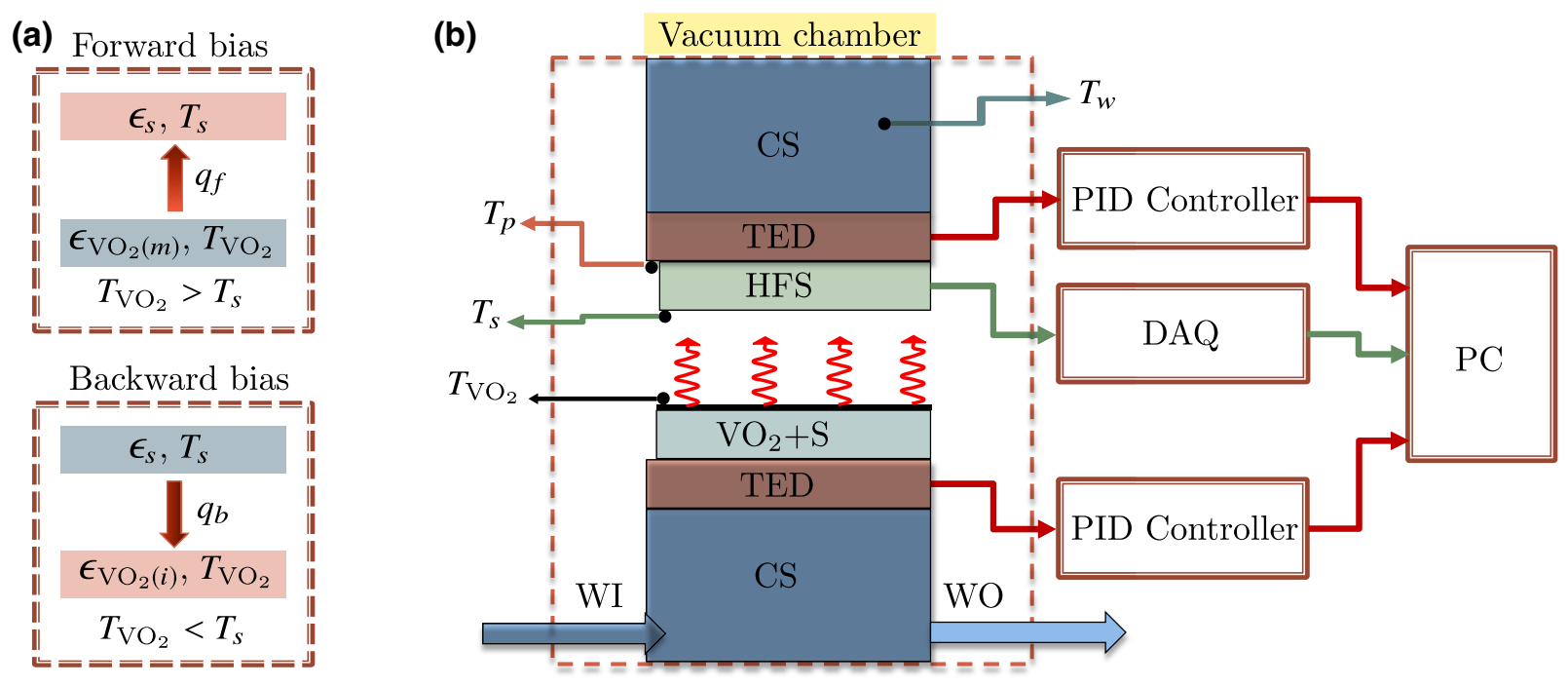

FIG. 1. Schemes of the (a) forward and backward configurations of the considered radiative thermal diode along with (b) its experimental setup. The direction of the radiative heat flux $q_{f}\left(q_{b}\right)$ is determined by the temperature condition $T_{\mathrm{VO}_{2}}>T_{s}\left(T_{\mathrm{VO}_{2}}<T_{s}\right)$. HFS, heat-flux sensor; $\mathrm{VO}_{2}+\mathrm{S}, \mathrm{VO}_{2}+$ substrate; TED, Peltier cell; CS, cooling system; WI, water inlet; WO, water outlet; DAQ, data-acquisition system; $T_{w}$, water temperature; $T_{p}$, Peltier temperature; $T_{s}$, HFS temperature; $T_{\mathrm{VO}_{2}}, \mathrm{VO}_{2}$ temperature. 
temperature $T_{a}=\left(T_{p}+T_{s}\right) / 2$ and the conversion from voltage to heat flux is determined by means of the calibration curve provided by the HFS manufacturer (greenTEG, $\mathrm{gSKIN}^{\circledR} \mathrm{XP}$ ). Data acquisition (DAQ) is performed by means of a Keysight USB system (U2300A).

\section{A. Temperature control}

Figure 2(a) shows the ability of our experimental system to maintain, increase or decrease the temperature of each terminal, when the set-point temperature $T_{\text {set }}$, controlled by a PID and represented by the red line, is modified. Initially, the system is taken to a steady state with $T_{\mathrm{VO}_{2}}=T_{s}=$ $40^{\circ} \mathrm{C}$, by fixing $T_{\text {set }}=40^{\circ} \mathrm{C}$ in both terminals. Next, $T_{\mathrm{VO}_{2}}$ is increased by means of the new set point $T_{\text {set }}=45^{\circ} \mathrm{C}$, which is reached by the $\mathrm{VO}_{2}$ film after a few minutes. This process is repeated in steps of $5^{\circ} \mathrm{C}$ up to $T_{\mathrm{VO}_{2}}=80^{\circ} \mathrm{C}$ (black line). Finally, the $\mathrm{VO}_{2}$ film is cooled down by repeating this procedure from 80 to $40{ }^{\circ} \mathrm{C}$ (black line). The temperature oscillations around the set points are smaller than $0.1^{\circ} \mathrm{C}$, which shows that the system response to the increase or decrease of the temperature of both terminals is smooth enough to ensure that $T_{\mathrm{VO}_{2}}$ and $T_{s}$ are well defined for accurately measuring the exchanged heat flux under steady-state conditions. In addition, the experimental data for the heat flux are recorded about 2 min after reaching the steady state and, therefore, the heat-flux values are independent of the heating and cooling speeds and time, as the typical response time of $\mathrm{VO}_{2}$ to a change of temperature is of a few milliseconds [38]. When fast heating rates are explored, large oscillations around the set point are observed before stabilization and there is no way to determine if the heat-flux values correspond to a heating or cooling process. On the other hand, during the whole experiment, the water temperature $T_{w}$ (purple line) raises around $1.6^{\circ} \mathrm{C}$, as a result of the work done by the water pump and the extraction of heat from the Peltier cells.
Figure 2(b) shows the typical heat flux $q$ as a function of time, for a $\mathrm{VO}_{2}$ film deposited on $c$-sapphire. $q$ is determined by means of $q=V / s$, where $V(\mu \mathrm{V})$ is the measured voltage and $s\left[\mu \mathrm{V} /\left(\mathrm{Wm}^{-2}\right)\right]$ is a temperature-dependent factor given by $s=21.03+\left(T_{a}-22.5\right) \times 0.0254$, as provided by the sensor manufacturer. The average heat flux thus obtained is $132.477 \pm 0.047 \mathrm{Wm}^{-2}$, which is then used to determine the rectification factor of the thermal diode under consideration.

\section{B. Calibration}

The measured heat flux $q$ as a function of temperature $T_{\mathrm{VO}_{2}}$ is shown in Fig. 3(a) for the heating-cooling cycle of a $\mathrm{VO}_{2}$ and $c$-sapphire sample between 30 and $90^{\circ} \mathrm{C}$. Note that during both the heating and cooling processes, $q$ varies almost linearly with $T_{\mathrm{VO}_{2}}$, for low and high enough values of $T_{\mathrm{VO}_{2}}$, as it typically occurs for materials with temperature-independent emissivities. By contrast, for intermediate temperatures between 62 and 68 ${ }^{\circ} \mathrm{C}\left(64\right.$ and $\left.58{ }^{\circ} \mathrm{C}\right)$, the heating (cooling) curve exhibits a significant drop (rise) of $118 \%(105 \%)$ due to the insulatorto-metal (metal-to-insulator) transition of the $\mathrm{VO}_{2}$ terminal. The temperature shift between the heating and cooling curves is partially due to the intrinsic thermal hysteresis of $\mathrm{VO}_{2}$, as reported in the literature [28]. During the whole cycle, the value of $T_{w}$ increases $1.9^{\circ} \mathrm{C}$, but it does not affect the sensor temperature $T_{s}$ [Fig. 3(b)], due to the use of a PID controller.

Note that even though the heat flux is driven by the MIT of $\mathrm{VO}_{2}$, its value does not reduce to zero for $T_{\mathrm{VO}_{2}}=$ $T_{s}=30^{\circ} \mathrm{C}$, as highlighted by the rectangle in Fig. 3(a). This indicates the presence of an nonradiative contribution detected by the HFS. This extra heat flux $q_{c}$ is attributed to the heat conduction between the HFS and water, and,
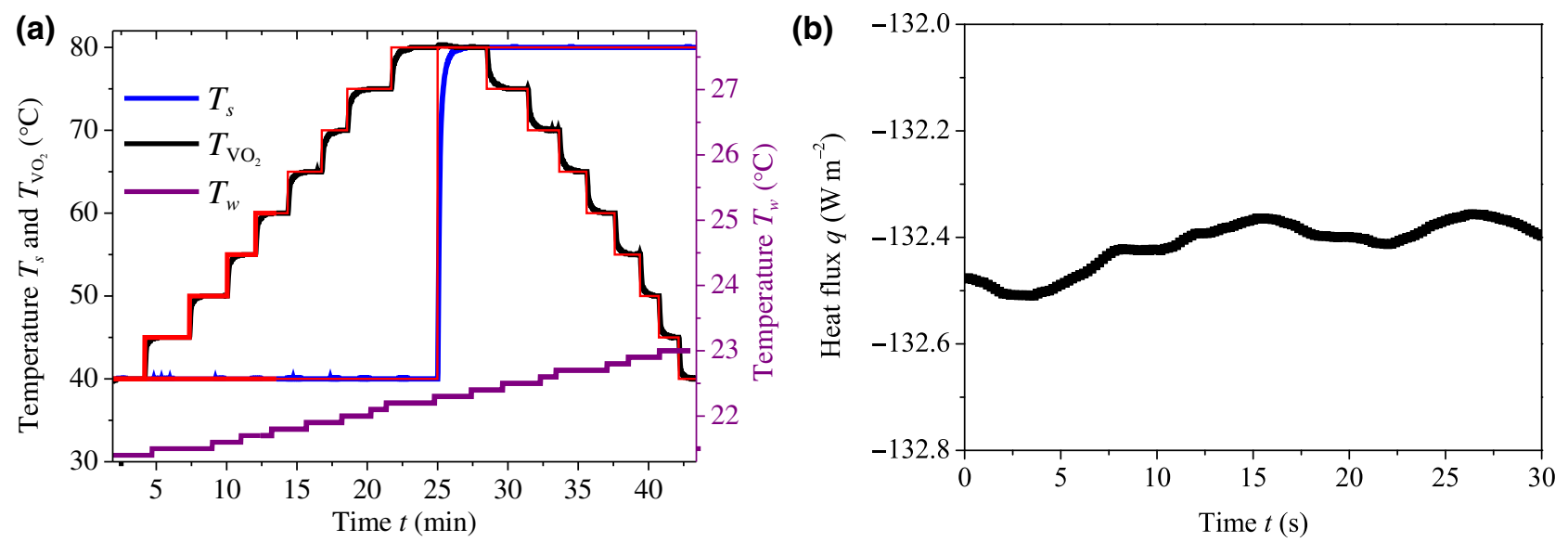

FIG. 2. Typical time evolution of the (a) temperature of the diode terminals $\left(T_{\mathrm{VO}_{2}} ; T_{s}\right)$ and water $T_{w}$ along with the (b) heat flux $q$ recorded for a $\mathrm{VO}_{2}$ film deposited on c-sapphire. Measurements in (b) are done for $T_{s}=70{ }^{\circ} \mathrm{C}$ and $T_{\mathrm{VO}_{2}}=30^{\circ} \mathrm{C}$. 

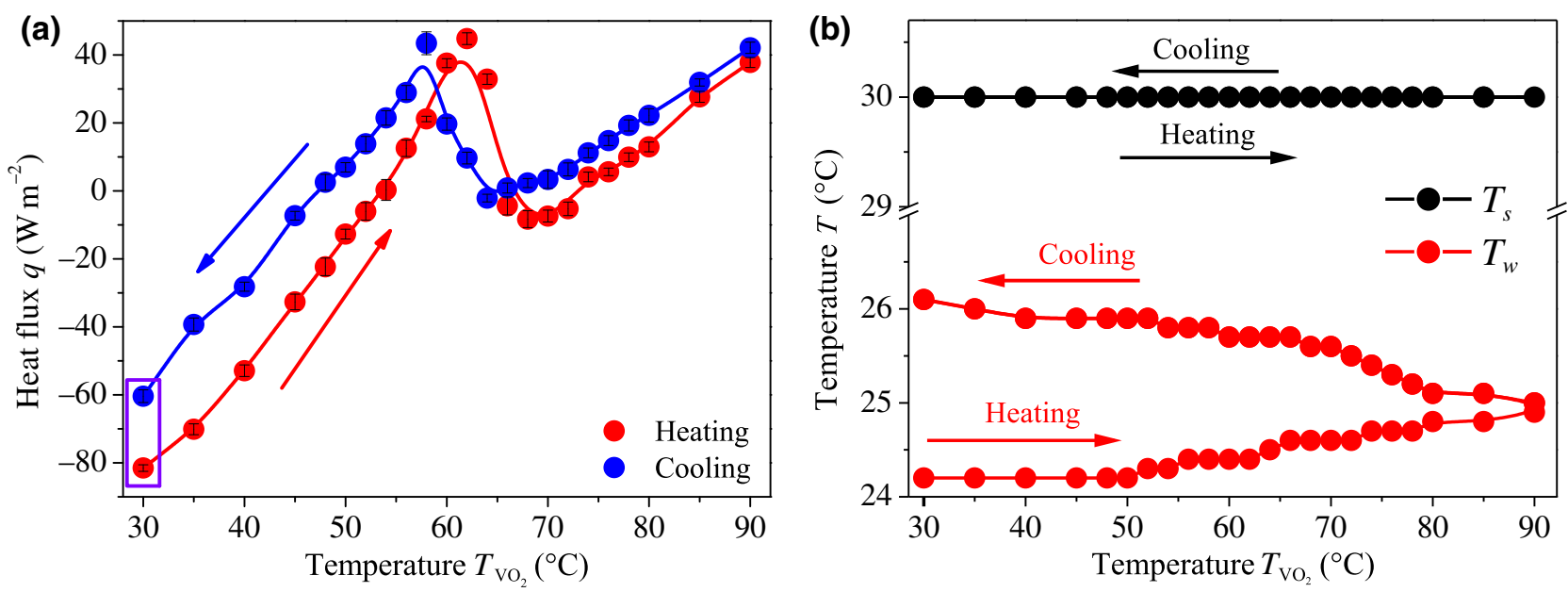

FIG. 3. (a) Heat flux and (b) temperatures $T_{s}$ and $T_{w}$ as functions of the $\mathrm{VO}_{2}$ terminal temperature, for a heating-cooling cycle between 30 and $90{ }^{\circ} \mathrm{C}$ of a $\mathrm{VO}_{2}$ film deposited over $c$-sapphire. Each experimental data point corresponds to the average of 100 measurements. Error bars included in (a) are very small and the solid lines joining the experimental points in both (a), (b) are only guides for the eye.

therefore, it can be determined by

$$
q_{c}=G\left(T_{s}-T_{w}\right),
$$

where $G\left(\mathrm{Wm}^{-2} \mathrm{~K}^{-1}\right)$ is an effective thermal conductance. Once the value of $G$ is determined by using the deviation of $q$ from zero (for $T_{\mathrm{VO}_{2}}=T_{s}=30^{\circ} \mathrm{C}$ ), the correction to each value of $q$ is applied by using the corresponding temperatures $\left(T_{w} ; T_{s}\right)$. This correction can be applied for all the range of temperatures covered, as can be seen in Sec. S1 within the Supplemental Material [39]. The heat-flux results thus obtained are shown in Sec. III, below.

\section{Sample preparation}

$\mathrm{VO}_{2}$ films are developed by using the pulsed-laser deposition (PLD) process inside an ultrahigh-vacuum chamber (nominal pressure $5 \times 10^{-8} \mathrm{mbar}$ ). An excimer nanosecond laser (LightMachinery IPEX-700, wavelength $\lambda=$ $248 \mathrm{~nm}$ ) is focused on a pure vanadium (99.95\%) metallic target under a reactive oxide atmosphere maintained at a pressure of $2200 \mathrm{~Pa}$. At a determined fluence of around $3 \mathrm{~J} \mathrm{~cm}^{-2}$, condensed matter is ablated and transferred to a substrate place in front of the target. Maintained at around $580^{\circ} \mathrm{C}$, the synthesized film grows on a substrate and pure phases of vanadium dioxide are obtained and crystallized, at these specific conditions.

Taking into account that the growth, microstructure, and quality of $\mathrm{VO}_{2}$ films are strongly determined by their substrate [28], in this study, three substrates ( $r$-sapphire, $c$-sapphire, and silicon) are selected to drastically modify their crystallographic orientations, grain sizes and shapes, and consequently their emissivity, as detailed in our previous work [28]. In this latter work, the temperature evolution of the emissivity of $\mathrm{VO}_{2}$ films is measured by means of the thermal wave-resonant cavity technique, whose working principle consists in detecting periodic temperature oscillations generated by a modulated infrared laser beam heating the sample. It is thus clear that the goal and methodology of our previous article [28] are totally different than the corresponding ones of the present work developed under steady-state conditions. Due to the relatively good atomic lattice matches [40], $c$-cut and $r$-cut sapphire substrates are chosen to promote $\mathrm{VO}_{2}$ synthesis, crystallization, orientation, and resulting properties $[41,42]$. The main characteristics of the three samples are summarized in Table I and their structural characterization can be seen in Sec. S2 within the Supplemental Material [39].

\section{RESULTS}

\section{A. Radiative heat flux and emissivity}

Figures 4(a)-4(c) show the temperature variations of the radiative heat flux $q$ exchanged by the HFS and a $\mathrm{VO}_{2}$ film deposited on a substrate of either $r$-sapphire or $c$-sapphire or silicon, respectively. Note that $q$ exhibits a clear hysteresis loop, whose characteristics depend strongly on the substrate, which is in a good agreement with previous works

TABLE I. Thicknesses and area of the substrates and fabricated $\mathrm{VO}_{2}$ films.

\begin{tabular}{lccc}
\hline \hline & \multicolumn{2}{c}{ Thickness } & \\
\cline { 2 - 3 } Sample & $\mathrm{VO}_{2}(\mathrm{~nm})$ & Substrate $(\mu \mathrm{m})$ & $\begin{array}{c}\text { Area } \\
\left(\mathrm{cm}^{2}\right)\end{array}$ \\
\hline $\mathrm{VO}_{2} / r$-sapphire & 300 & 430 & 2.25 \\
$\mathrm{VO}_{2} / c$-sapphire & 200 & 500 & 2.25 \\
$\mathrm{VO}_{2} /$ silicon & 200 & 525 & 2.25 \\
\hline \hline
\end{tabular}


(a)

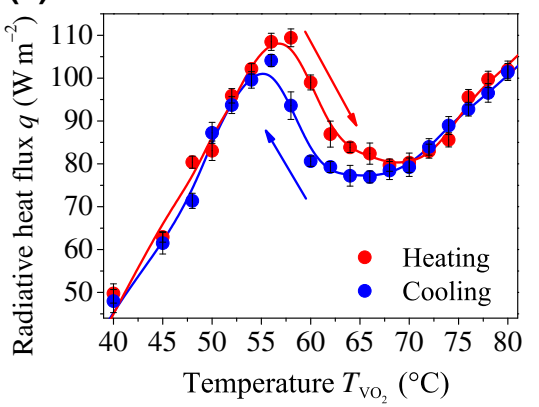

(b)

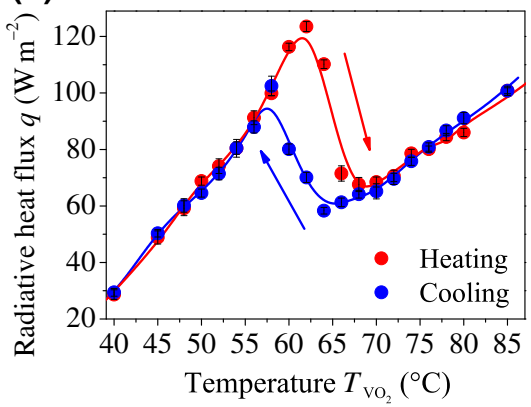

(c)

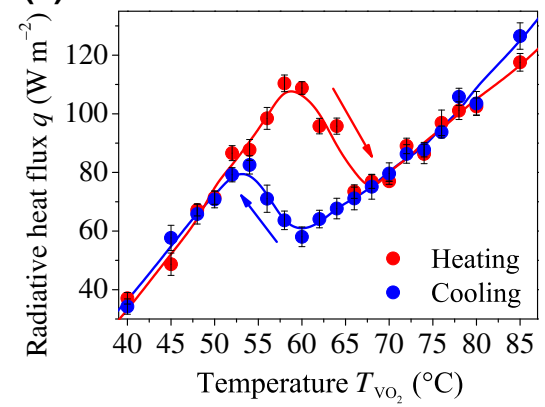

FIG. 4. Radiative heat flux as a function of $T_{\mathrm{VO}_{2}}$ for the heating-cooling cycle of a $\mathrm{VO}_{2}$ film deposited on (a) $r$-sapphire, (b) $c$ sapphire, and (c) silicon. Red and blue experimental points stand for heating and cooling processes with $T_{s}=30^{\circ} \mathrm{C}$, respectively. Each point corresponds to the average of 100 measurements, which are used to determine the relatively small error bars. Solid lines are just guides to the eye.

$[24,43,44]$. Silicon yields the widest loop, while $r$-sapphire the thinnest one; and they represent the fingerprints of the MIT of $\mathrm{VO}_{2}$. The behavior of $q$ is driven by the emissivity and geometry of both terminals, as established by the Stefan-Boltzmann law [45]

$$
q=\epsilon_{\mathrm{eff}} \sigma\left(T_{\mathrm{VO}_{2}}^{4}-T_{s}^{4}\right),
$$

where $\sigma=5.67 \times 10^{-8} \mathrm{Wm}^{-2} \mathrm{~K}^{-4}$ is the Stefan-Boltzmann constant and the effective emissivity $\epsilon_{\text {eff }}=$ $\left[\left(\epsilon_{\mathrm{VO}_{2}}^{-1}-1\right) / A_{\mathrm{VO}_{2}}+1 /\left(A_{\mathrm{VO}_{2}} F\right)+\left(\epsilon_{s}^{-1}-1\right) / A_{s}\right]^{-1}$, with $\epsilon_{s}=0.78$ and $F=0.36$ being the HFS emissivity and view factor [46], respectively. Details of the view-factor calculation are given in Sec. S3 within the Supplemental Material [39]. Equation (3) thus allows determining the $\mathrm{VO}_{2}$ emissivity $\epsilon_{\mathrm{VO}_{2}}$ shown in Fig. 5, by using the heatflux values displayed in Fig. 4. One can see that the critical temperature $\left(T_{c}\right)$, hysteresis width $(\Delta H)$ and slope of the heating curve $(\beta)$ are different for the three $\mathrm{VO}_{2}$ films and their values are summarized in Table II. This is reasonable, as these parameters are typically determined by the substrate [40,47-49] and the film thickness [40,48-50]. In particular, note that the samples $\mathrm{VO}_{2}$ and silicon, and
$\mathrm{VO}_{2}$ and $c$-sapphire have hysteresis widths 4 and 1.5 times greater with that of $\mathrm{VO}_{2}$ and $r$-sapphire, which exhibits the largest emissivity variation $(0.63)$ between its insulating and metallic phases. The temperature evolution of the emissivities obtained for the three $\mathrm{VO}_{2}$ films are consistent with those reported in the literature $[28,51]$ and their values in the insulating phase are close to the corresponding ones of their substrates, as $\mathrm{VO}_{2}$ is fairly transparent to the infrared radiation in this latter phase [34]. These results ensure that the radiative heat flux measured by our experimental setup can be considered reliable. On the other hand, note that $\Delta H$ and $\beta$ have an inverse relationship, that is to say, $\mathrm{VO}_{2}$ samples with larger hysteresis widths have slower phase transitions, in agreement with previous works $[28,36,40]$. These features are fundamental for the development of thermal memory devices $[13,44]$ and thermal logic gates [11], which require large and small $\Delta H$ values, respectively.

\section{B. Rectification factors}

The radiative heat flux $q$ measured for the forward $\left(T_{\mathrm{VO}_{2}}>T_{s}=45^{\circ} \mathrm{C}\right)$ and backward $\left(T_{\mathrm{VO}_{2}}<T_{s}=85^{\circ} \mathrm{C}\right)$ configurations between the HFS and a $\mathrm{VO}_{2}$ film deposited (a)

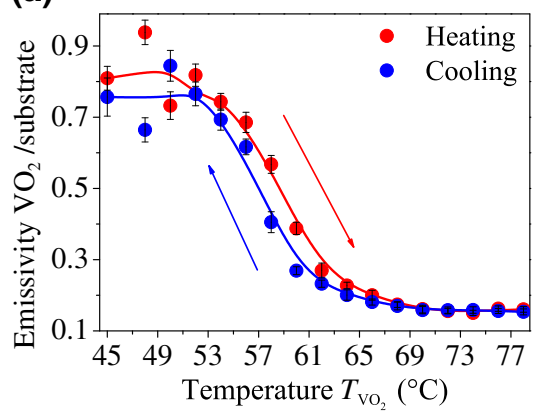

(b)

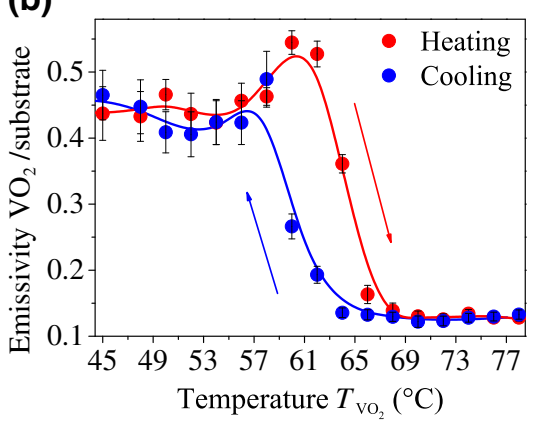

(c)

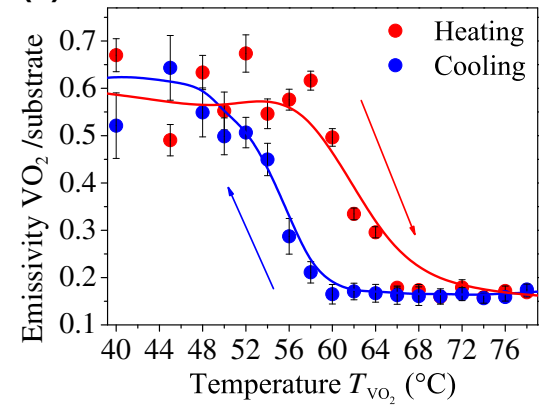

FIG. 5. Temperature evolution of the emissivity determined through Eq. (3) for a $\mathrm{VO}_{2}$ film deposited on (a) $r$-sapphire, (b) $c$ sapphire, and (c) silicon. Solid lines represent visual guides to the eye. 
TABLE II. MIT characteristics in the emissivity of $\mathrm{VO}_{2}$ films grown on a $r$-sapphire, $c$-sapphire, and silicon substrate. Vertical arrow, for the slope $\beta$ stand for the heating process.

\begin{tabular}{lcccc}
\hline \hline Sample & $\Delta \epsilon_{\mathrm{VO}_{2}}$ & $\Delta H\left({ }^{\circ} \mathrm{C}\right)$ & $T c\left({ }^{\circ} \mathrm{C}\right)$ & $\beta\left[{ }^{\circ} \mathrm{C}^{-1}(\uparrow)\right]$ \\
\hline $\mathrm{VO}_{2} / r$-sapphire & 0.63 & 1.7 & 59 & 0.08 \\
$\mathrm{VO}_{2} / c$-sapphire & 0.33 & 2.5 & 62 & 0.05 \\
$\mathrm{VO}_{2} /$ silicon & 0.43 & 7 & 60 & 0.03 \\
\hline \hline
\end{tabular}

on $r$-sapphire, $c$-sapphire, and silicon is, respectively, shown in Figs. 6(a)-6(c), as a function of the temperature difference $\left(\Delta T=T_{\mathrm{VO}_{2}}-T_{s}\right)$ between the diode terminals. The experimental data are obtained by fixing the sensor temperature $T_{s}=45^{\circ} \mathrm{C}$ and increasing $T_{\mathrm{VO}_{2}}$ from 35 to $90^{\circ} \mathrm{C}$. Then, $T_{s}$ is raised to $85^{\circ} \mathrm{C}$ and $T_{\mathrm{VO}_{2}}$ decreased from 90 to $35^{\circ} \mathrm{C}$. This detailed process allowed us to clearly identify the $\mathrm{VO}_{2}$ phase transition and any anomaly on the measured heat flux. Taking into account that the rectification factor of a thermal diode is defined in terms of the radiative heat fluxes obtained by interchanging the temperatures of the diode terminals, as established by Eq. (1), Fig. 6 displays the real operation of a thermal diode for the pair of temperatures $(45 ; 85){ }^{\circ} \mathrm{C}$ (purple points) of its terminals. This procedure is also repeated for other pairs of temperatures to determine the diode rectification factor. Note that the magnitude of $q$ increases with $|\Delta T|$, such that its values in the backward configuration are greater than the corresponding ones in the forward bias, for a given $|\Delta T|$. This is reasonable, given that the backward configuration is driven by the $\mathrm{VO}_{2}$ emissivity in its insulating phase, which is higher than that in the metallic one, as shown in Fig. 5. Samples grown on silicon and $c$-sapphire yield a common maximum rectification factor of $50 \%$, which increases to $61 \%$ for the sample deposited on $r$-sapphire. This latter value surpasses the rectification factor of $56 \%$ reported by Fiorino et al. [23] for a near-field thermal diode based on $\mathrm{VO}_{2}$ and operating with a temperature difference of $70^{\circ} \mathrm{C}$ between its terminals. Thus, our best result not only optimizes the rectification factor, but also reduces to $85-45=40^{\circ} \mathrm{C}$ the temperature difference required to obtain it. This significant reduction is also present for the rectification of far-field heat currents previously reported by Ito et al. [24] with a similar rectification factor. In this latter work, the optimization of the rectification factor is performed by testing $\mathrm{VO}_{2}$ and $W$-doped $\mathrm{VO}_{2}$ films deposited on silicon, while in our present work, we do it by changing the substrate ( $r$-sapphire, $c$-sapphire, and silicon) of pure $\mathrm{VO}_{2}$ films. In addition, the methodology used by Ito et al. [24] is different than ours in the following aspects. (i) The HFS is placed between the $\mathrm{VO}_{2}$ substrate and the thermoelectric device used to control the temperature and hence it can only measure conductive heat fluxes. By contrast, our HFS acts as a diode terminal and directly measures the radiative heat flux exchanged with the other diode terminal. (ii) While the calibration of our experimental setup is done with the sample to be tested, Ito et al. [24] performed the calibration using fused quartz and measurements with their $\mathrm{VO}_{2}$ samples. This replacement of the material to calibrate by the one to be tested implies variations of the vacuum conditions (opening of the vacuum chamber) and the modification of the interface thermal resistance between the sample and the thermoelectric device, which could generate uncertainties on the measurements. (iii) The theoretical description of the measured heat flux is based on the fluctuational electrodynamics, whereas we apply the Stefan-Boltzmann equation that allows us to retrieve the emissivity of our $\mathrm{VO}_{2}$ samples, as shown in Fig. 5. Even though both formalisms are correct for the considered far-field radiation, the advantage of Boltzmann approach is that it can be applied to derive an analytical expression [see Eq. (4)] for the optimal rectification factor. Based on these significant differences and the remarkable reduction of the temperature difference (from 70 to $40^{\circ} \mathrm{C}$ ), the present methodology and results are expected to be useful for both optimizing (a)

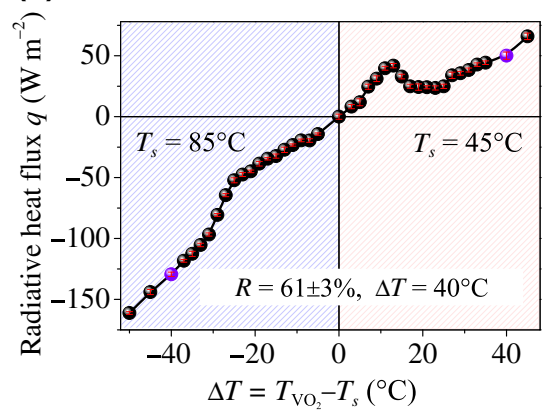

(b)

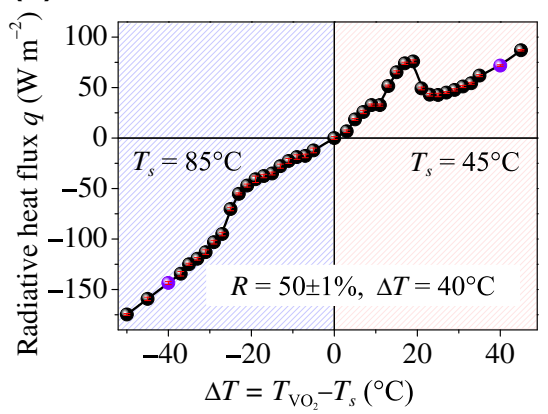

(c)

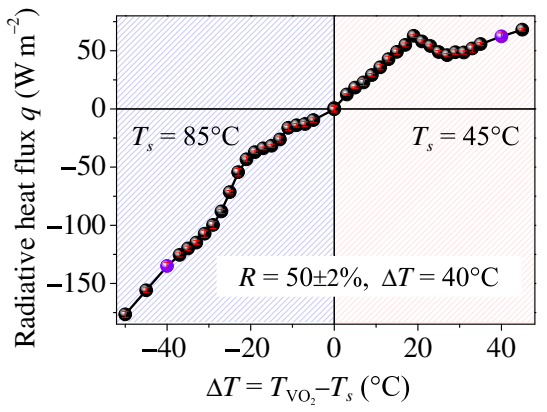

FIG. 6. Measured radiative heat flux between the HFS and a $\mathrm{VO}_{2}$ film deposited on (a) $r$-sapphire, (b) $c$-sapphire, and (c) silicon, as a function of the temperature difference $\Delta T=T_{\mathrm{VO}_{2}}-T_{s}$. The given rectification factors are obtained by means of Eq. (1) for $\Delta T=40^{\circ} \mathrm{C}$ ( $q$ values highlighted in purple). The bluish and reddish backgrounds stand for the backward and forward configuration, respectively. 

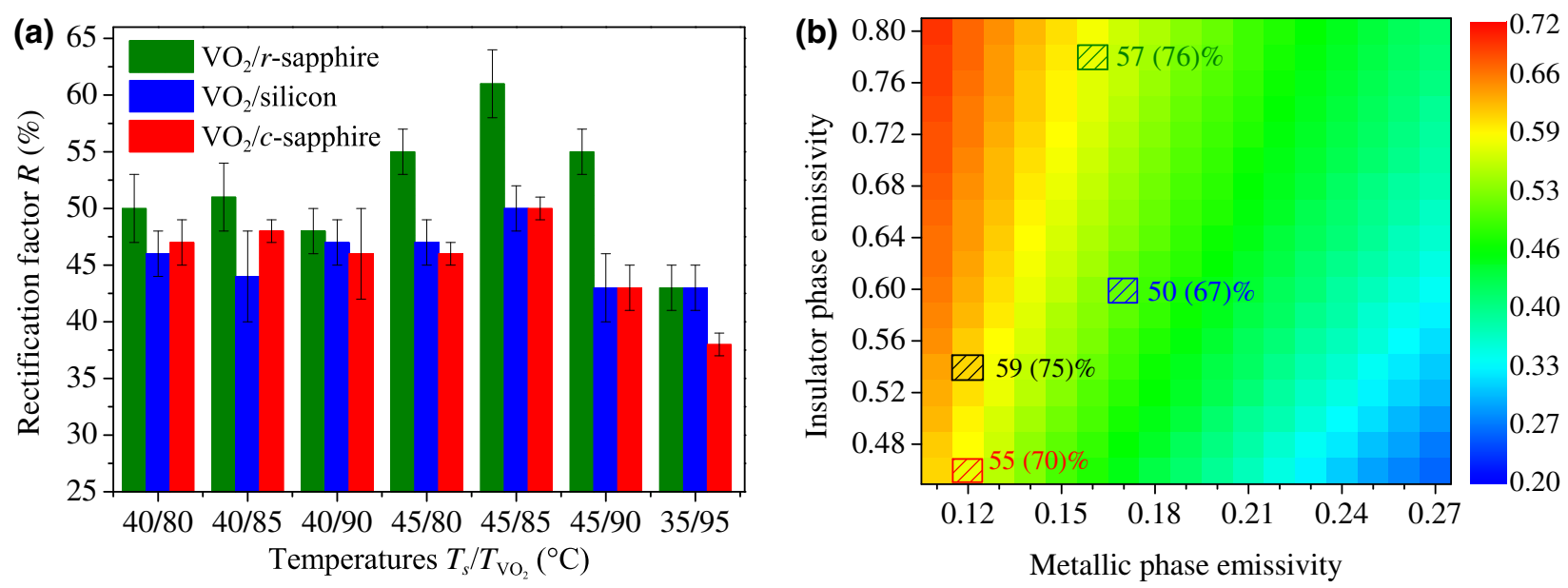

FIG. 7. (a) Summary of the rectification factors obtained by means of the experimental data shown in Fig. 6 and Eq. (1), for the three $\mathrm{VO}_{2}$ samples and different temperature pairs $\left(T_{s} ; T_{\mathrm{VO}_{2}}\right)$ of the diode terminals. (b) Optimal rectification factor predicted by Eq. (4) for different values of the emissivities of $\mathrm{VO}_{2}$ in its metallic and insulating phases. The green, red, and blue squares stand for the rectification factors determined with the data reported in Fig. 5 for a $\mathrm{VO}_{2}$ film deposited on $r$-sapphire, $c$-sapphire, and silicon, respectively. The data in parenthesis stand for an ideal thermal diode made up of two infinite parallel terminals. The black square represents the rectification factor $(59 \%)$ obtained for the emissivity peak of the $\mathrm{VO}_{2}$ and $c$-sapphire sample at $61{ }^{\circ} \mathrm{C}$.

the rectification factor of a radiative thermal diode and reducing the temperature difference of its terminals.

Figure 7(a) shows a summary of the highest rectification factors obtained for each $\mathrm{VO}_{2}$ sample and different temperature pair $\left(T_{s} ; T_{\mathrm{VO}_{2}}\right)$ of the diode terminals. In order to capitalize on the $\mathrm{VO}_{2}$ phase transition, the values of both $T_{s}$ and $T_{\mathrm{VO}_{2}}$ are conveniently chosen outside it. Note that the $\mathrm{VO}_{2}$ film grown on $r$-sapphire allows obtaining rectification factors higher than the corresponding ones yielded by the other two samples, for each temperature pair. The highest rectification is obtained for $\left(T_{s} ; T_{\mathrm{VO}_{2}}\right)=$ $(45 ; 85)^{\circ} \mathrm{C}$, while the lowest one appears for $\left(T_{s} ; T_{\mathrm{VO}_{2}}\right)=$ $(35 ; 95){ }^{\circ} \mathrm{C}$. Taking into account that the values of these latter temperatures are well outside the $\mathrm{VO}_{2}$ phase transition, this fact indicates that the maximum rectification factor is strongly determined by the $\mathrm{VO}_{2}$ emissivity in its insulating $\left[\epsilon_{\mathrm{VO}_{2}(i)}\right]$ and metallic $\left[\epsilon_{\mathrm{VO}_{2}(m)}\right]$ phases. In fact, according to Eqs. (1) and (3), one can show that the optimal (maximum) rectification factor is given by

$$
R_{o}=\frac{1-\epsilon_{\mathrm{VO}_{2}(m)} / \epsilon_{\mathrm{VO}_{2}(i)}}{1+\epsilon_{\mathrm{VO}_{2}(m)}\left[A_{\mathrm{VO}_{2}}\left(\epsilon_{s}^{-1}-1\right) / A_{s}+F^{-1}-1\right]} .
$$

Note that $R_{o}$ increases with the view factor $(F=1$ and $A_{\mathrm{VO}_{2}} / A_{s}=1$ for the ideal case of infinite parallel plates), emissivity ratio $\epsilon_{\mathrm{VO}_{2}(i)} / \epsilon_{\mathrm{VO}_{2}(m)}$ and sensor emissivity $\epsilon_{s}$, such as higher rectifications are obtained for lower emissivities $\epsilon_{\mathrm{VO}_{2}(m)}$ of $\mathrm{VO}_{2}$ in its metallic phase. Equation (4) thus establishes that the best $\mathrm{VO}_{2}$ sample to optimize the rectification factor of a radiative diode is the one with not only the highest emissivity contrast between its insulating and metallic phases, but also with the lowest emissivity
$\epsilon_{\mathrm{VO}_{2}(m)}$. Among the three $\mathrm{VO}_{2}$ samples considered in this work, both of these latter conditions are fulfilled by the $\mathrm{VO}_{2}$ film deposited on $r$-sapphire, as shown in Fig. 5(a). The other two samples exhibit relatively large hysteresis widths, which are required for improving the performance of thermal memory devices $[13,44]$.

Figure 7(b) shows the predictions of Eq. (4) for the emissivities in the metallic and insulator states of the three $\mathrm{VO}_{2}$ samples displayed in Figs. 5. Optimal rectifications of $57(76) \%, 55(70) \%$ and $50(67) \%$ are obtained for the $\mathrm{VO}_{2}$ films deposited on $r$-sapphire, $c$-sapphire, and silicon, respectively. The numbers in parenthesis stand for the rectification factors of an ideal thermal diode made up of two infinite parallel terminals. Note that even though the emissivity variation of the $\mathrm{VO}_{2}$ and $c$-sapphire sample between its insulating and metallic phases is nearly half compared to that obtained for the $\mathrm{VO}_{2} / r$-sapphire one, the optimal rectification factors determined for both samples are pretty much the same, due to the relatively low emissivity $\epsilon_{\mathrm{VO}_{2}(m)}$ of the sample grown on $c$-sapphire. These results are in good agreement with the ones shown in Fig. 7 (a) and reported in the literature $[10,28]$. The slight differences between the results displayed in Figs. 7(a) and 7(b) is attributed to the fluctuations of the $\mathrm{VO}_{2}$ emissivity in its insulating phase, which can lead to variations of around $5 \%$ on $R_{o}$. This consistence of the optimal rectification factors obtained by means of the heat flux and emissivity values confirms that to the $\mathrm{VO}_{2}$ film grown on $r$-sapphire is better than the other two samples to operate a radiative thermal diode. Finally, in future works, the optimal rectification factor could be enhanced by $3 \%$, increasing the sensor emissivity from 0.78 to 0.95 . In addition, we note that the emissivity peak exhibited by $c$-sapphire (at $61^{\circ} \mathrm{C}$ ) 
could also be used to increase $R_{o}$ by $3 \%$, as shown by the black square in Fig. 7(b).

\section{CONCLUSIONS}

We experimentally demonstrate the thermal rectification of far-field heat currents by exploiting the metal-insulator transition of $\mathrm{VO}_{2}$. This is achieved by measuring the radiative heat flux between a heat-flux sensor and a $\mathrm{VO}_{2}$ film deposited on either $r$-sapphire or $c$-sapphire or silicon. The highest rectification factor of $61 \%$ is obtained with $r$ sapphire, which is among the highest values reported in the literature with the lowest temperature difference $\left(40^{\circ} \mathrm{C}\right)$ between the diode terminals. This experimental value for the rectification factor is consistent with the theoretical prediction of an analytical expression that has been derived for the maximum rectification factor, as a function of the $\mathrm{VO}_{2}$ emissivity in the metallic and insulating phases, sensor emissivity, and geometrical parameters. The obtained results thus show that the rectification factor of radiative diodes can be enhanced, while reducing the temperature difference of their terminals, by not only increasing the emissivity variation between the insulating and metallic phases of $\mathrm{VO}_{2}$ films deposited on $r$-sapphire, but also decreasing their emissivity in the metallic phase.

\section{ACKNOWLEDGMENTS}

This work was partially supported by the projects 251882 "Investigación Científica Básica 2015", SREAMEXCID-2016-1-278320, and ANR-19-CE09-0005 "EPolariton". J.C.-E. thanks CONACYT for his master scholarship (10.13039/501100003141). The authors are grateful to J. Bante-Guerra for his technical assistance.

[1] B. Li, L. Wang, and G. Casati, Thermal Diode: Rectification of Heat Flux, Phys. Rev. Lett. 93, 184301 (2004).

[2] C. R. Otey, W. T. Lau, and S. Fan, Thermal Rectification through Vacuum, Phys. Rev. Lett. 104, 154301 (2010).

[3] P. Ben-Abdallah and S.-A. Biehs, Phase-change radiative thermal diode, Appl. Phys. Lett. 103, 191907 (2013).

[4] J. Huang, Q. Li, Z. Zheng, and Y. Xuan, Thermal rectification based on thermochromic materials, Int. J. Heat Mass Transf. 67, 575 (2013).

[5] Y. Yang, S. Basu, and L. Wang, Radiation-based near-field thermal rectification with phase transition materials, Appl. Phys. Lett. 103, 163101 (2013).

[6] P. Ben-Abdallah and S.-A. Biehs, Near-Field Thermal Transistor, Phys. Rev. Lett. 112, 044301 (2014).

[7] K. Joulain, J. Drevillon, Y. Ezzahri, and J. OrdonezMiranda, Quantum Thermal Transistor, Phys. Rev. Lett. 116, 1 (2016).

[8] J. Ordonez-Miranda, Y. Ezzahri, J. Drevillon, and K. Joulain, Transistorlike Device for Heating and Cooling Based on the Thermal Hysteresis of $\mathrm{VO}_{2}$, Phys. Rev. Appl. 6, 054003 (2016).
[9] H. Prod'homme, J. Ordonez-Miranda, Y. Ezzahri, J. Drevillon, and $\mathrm{K}$. Joulain, Optimized thermal amplification in a radiative transistor, J. Appl. Phys. 119, 194502 (2016).

[10] K. Joulain, Y. Ezzahri, J. Drevillon, and P. Ben-Abdallah, Modulation and amplification of radiative far field heat transfer: Towards a simple radiative thermal transistor, Appl. Phys. Lett. 106, 133505 (2015).

[11] L. Wang and B. Li, Thermal Logic Gates: Computation with Phonons, Phys. Rev. Lett. 99, 177208 (2007).

[12] P. Ben-Abdallah and S.-A. Biehs, Towards Boolean operations with thermal photons, Phys. Rev. B 94, 241401 (2016).

[13] L. Wang and B. Li, Thermal Memory: A Storage of Phononic Information, Phys. Rev. Lett. 101, 267203 (2008).

[14] V. Kubytskyi, S.-A. Biehs, and P. Ben-Abdallah, Radiative Bistability and Thermal Memory, Phys. Rev. Lett. 113, 074301 (2014).

[15] C. Starr, The copper oxide rectifier, J. Appl. Phys. 7, 15 (1936).

[16] E. Nefzaoui, J. Drevillon, Y. Ezzahri, and K. Joulain, Simple far-field radiative thermal rectifier using Fabry-Perot cavities based infrared selective emitters, Appl. Opt. 53, 3479 (2014).

[17] W. Kobayashi, D. Sawaki, T. Omura, T. Katsufuji, Y. Moritomo, and I. Terasaki, Thermal rectification in the vicinity of a structural phase transition, Appl. Phys. Express 5, 027302 (2012).

[18] E. Pallecchi, Z. Chen, G. E. Fernandes, Y. Wan, J. H. Kim, and $\mathrm{J} . \mathrm{Xu}, \mathrm{A}$ thermal diode and novel implementation in a phase-change material, Mater. Horizons 2, 125 (2015).

[19] N. Li, J. Ren, L. Wang, G. Zhang, P. Hänggi, and B. Li, Colloquium: Phononics: Manipulating heat flow with electronic analogs and beyond, Rev. Mod. Phys. 84, 1045 (2012).

[20] N. Roberts and D. Walker, A review of thermal rectification observations and models in solid materials, Int. J. Therm. Sci. 50, 648 (2011).

[21] T. Villeneuve, M. Boudreau, and G. Dumas, The thermal diode and insulating potentials of a vertical stack of parallelogrammic air-filled enclosures, Int. J. Heat Mass Transf. 108, 2060 (2017).

[22] A. Pugsley, A. Zacharopoulos, J. Deb Mondol, and M. Smyth, Theoretical and experimental analysis of a horizontal planar liquid-vapour Thermal diode (PLVTD), Int. J. Heat Mass Transf. 144, 118660 (2019).

[23] A. Fiorino, D. Thompson, L. Zhu, R. Mittapally, S.-A. Biehs, O. Bezencenet, N. El-Bondry, S. Bansropun, P. BenAbdallah, E. Meyhofer, and P. Reddy, A thermal diode based on nanoscale thermal radiation, ACS Nano 12, 5774 (2018).

[24] K. Ito, K. Nishikawa, H. Iizuka, and H. Toshiyoshi, Experimental investigation of radiative thermal rectifier using vanadium dioxide, Appl. Phys. Lett. 105, 253503 (2014).

[25] A. Ghanekar, G. Xiao, and Y. Zheng, High contrast far-field radiative thermal diode, Sci. Rep. 7, 6339 (2017).

[26] D. Polder and M. Van Hove, Theory of radiative heat transfer between closely spaced bodies, Phys. Rev. B 4, 3303 (1971).

[27] P. J. van Zwol, K. Joulain, P. Ben-Abdallah, and J. Chevrier, Phonon polaritons enhance near-field thermal 
transfer across the phase transition of $\mathrm{VO}_{2}$, Phys. Rev. B 84, 161413 (2011).

[28] C. L. Gomez-Heredia, J. A. Ramirez-Rincon, J. OrdonezMiranda, O. Ares, J. J. Alvarado-Gil, C. Champeaux, F. Dumas-Bouchiat, Y. Ezzahri, and K. Joulain, Thermal hysteresis measurement of the $\mathrm{VO}_{2}$ emissivity and its application in thermal rectification, Sci. Rep. 8, 8479 (2018).

[29] G. Hamaoui, N. Horny, C. L. Gomez-Heredia, J. A. Ramirez-Rincon, J. Ordonez-Miranda, C. Champeaux, F. Dumas-Bouchiat, J. J. Alvarado-Gil, Y. Ezzahri, K. Joulain, and M. Chirtoc, Thermophysical characterisation of $\mathrm{VO}_{2}$ thin films hysteresis and its application in thermal rectification, Sci. Rep. 9, 8728 (2019).

[30] A. S. Barker, H. W. Verleur, and H. J. Guggenheim, Infrared Optical Properties of Vanadium Dioxide Above and Below the Transition Temperature, Phys. Rev. Lett. 17, 1286 (1966).

[31] A. Zylbersztejn and N. F. Mott, Metal-insulator transition in vanadium dioxide, Phys. Rev. B 11, 4383 (1975).

[32] D.-W. Oh, C. Ko, S. Ramanathan, and D. G. Cahill, Thermal conductivity and dynamic heat capacity across the metal-insulator transition in thin film $\mathrm{VO}_{2}$, Appl. Phys. Lett. 96, 151906 (2010).

[33] J. Ordonez-Miranda, Y. Ezzahri, K. Joulain, J. Drevillon, and J. J. Alvarado-Gil, Modeling of the electrical conductivity, thermal conductivity, and specific heat capacity of $\mathrm{VO}_{2}$, Phys. Rev. B 98, 075144 (2018).

[34] H. S. Choi, J. S. Ahn, J. H. Jung, T. W. Noh, and D. H. Kim, Mid-infrared properties of a $\mathrm{VO}_{2}$ film near the metal-insulator transition, Phys. Rev. B 54, 4621 (1996).

[35] M. M. Qazilbash, M. Brehm, G. O. Andreev, A. Frenzel, P.-C. Ho, B.-G. Chae, B.-J. Kim, S. J. Yun, H.-T. Kim, A. V. Balatsky, O. G. Shpyrko, M. B. Maple, F. Keilmann, and D. N. Basov, Infrared spectroscopy and nano-imaging of the insulator-to-metal transition in vanadium dioxide, Phys. Rev. B 79, 075107 (2009).

[36] J. A. Ramirez-Rincon, C. L. Gomez-Heredia, A. Corvisier, J. Ordonez-Miranda, T. Girardeau, F. Paumier, C. Champeaux, F. Dumas-Bouchiat, Y. Ezzahri, K. Joulain, O. Ares, and J. J. Alvarado-Gil, Thermal hysteresis measurement of the $\mathrm{VO}_{2}$ dielectric function for its metal-insulator transition by visible-IR ellipsometry, J. Appl. Phys. 124, 195102 (2018).

[37] G. Wehmeyer, T. Yabuki, C. Monachon, J. Wu, and C. Dames, Thermal diodes, regulators, and switches: Physical mechanisms and potential applications, Appl. Phys. Rev. 4, 041304 (2017).

[38] H. Wang, X. Yi, S. Chen, and X. Fu, Fabrication of vanadium oxide micro-optical switches, Sens. Actuator A Phys. 122, 108 (2005).
[39] See Supplemental Material at http://ink.aps.org/supple mental/10.1103/PhysRevApplied.14.034023 for the calibration, structural characterization, and view-factor calculation.

[40] T.-H. Yang, R. Aggarwal, A. Gupta, H. Zhou, R. J. Narayan, and J. Narayan, Semiconductor-metal transition characteristics of $\mathrm{VO}_{2}$ thin films grown on c- and r-sapphire substrates, J. Appl. Phys. 107, 053514 (2010).

[41] K. D. Rogers, An X-ray diffraction study of semiconductor and metallic vanadium dioxide, Powder Diffr. 8, 240 (1993).

[42] Y. Zhao, J. Hwan Lee, Y. Zhu, M. Nazari, C. Chen, H. Wang, A. Bernussi, M. Holtz, and Z. Fan, Structural, electrical, and terahertz transmission properties of $\mathrm{VO}_{2}$ thin films grown on c-, r-, and m-plane sapphire substrates, J. Appl. Phys. 111, 053533 (2012).

[43] D. M. Bierman, A. Lenert, M. A. Kats, Y. Zhou, S. Zhang, M. De La Ossa, S. Ramanathan, F. Capasso, and E. N. Wang, Radiative Thermal Runaway Due to NegativeDifferential Thermal Emission Across a Solid-Solid Phase Transition, Phys. Rev. Appl. 10, 021001 (2018).

[44] K. Ito, K. Nishikawa, and H. Iizuka, Multilevel radiative thermal memory realized by the hysteretic metal-insulator transition of vanadium dioxide, Appl. Phys. Lett. 108, 053507 (2016).

[45] B. Bird, W. Stewart, and E. Lightfoot, Transport Phenomena (John Wiley \& Sons, New York, 2007), 2nd ed.

[46] J. R. Ehlert and T. F. Smith, View factors for perpendicular and parallel rectangular plates, J. Thermophys. Heat Transf. 7, 173 (1993).

[47] S. Saitzek, F. Guinneton, G. Guirleo, L. Sauques, K. Aguir, and J. R. Gavarri, $\mathrm{VO}_{2}$ thin films deposited on silicon substrates from $\mathrm{V}_{2} \mathrm{O}_{5}$ target: Limits in optical switching properties and modeling, Thin Solid Films 516, 891 (2008).

[48] M.-W. Kim, W.-G. Jung, T.-S. Hyun-Cho, S.-J. Bae, J.-S. Chang, Jang, W.-K. Hong, and B.-J. Kim, Substratemediated strain effect on the role of thermal heating and electric field on metal-insulator transition in vanadium dioxide nanobeams, Sci. Rep. 5, 10861 (2015).

[49] F. Guinneton, L. Sauques, J.-C. Valmalette, F. Cros, and J.-R. Gavarri, Optimized infrared switching properties in thermochromic vanadium dioxide thin films: Role of deposition process and microstructure, Thin Solid Films 446, 287 (2004).

[50] R. L. Voti, M. C. Larciprete, G. Leahu, C. Sibilia, and M. Bertolotti, Optimization of thermochromic $\mathrm{VO}_{2}$ based structures with tunable thermal emissivity, J. Appl. Phys. 112, 034305 (2012).

[51] M. A. Kats, R. Blanchard, S. Zhang, P. Genevet, C. Ko, S. Ramanathan, and F. Capasso, Vanadium Dioxide as a Natural Disordered Metamaterial: Perfect Thermal Emission and Large Broadband Negative Differential Thermal Emittance, Phys. Rev. X 3, 041004 (2013). 